\title{
Nutrition\& Metabolism
}

Aadil, N. 296

Abbey, M. 269

Abdella, N. 329

Akanji, A.O. 1

Alarcón, R. 28

Allard, J. 381

Aller, R. 141

Alsaeid, M. 329

Al-Shayji, I.A.R. 1

Al-Sheikh, N. 329

Amer, M. 343

Amouyel, P. 43

An, H.-J. 398

Antal, M. 307

Arruda, S.F. 288

Arveiler, D. 43

Asadifar, M. 79

Astheimer, L.B. 67

Azuma, A. 390

Baek, S.-H. 398

Baillet-Blanco, L. 409

Balajti, A. 307

Bannai, S. 348

Barthes, N. 409

Bernhardi, R. von 28

Bicudo Pereira Costa-Rosa, L.F. 90

Bingham, A. 43

Biró, L. 307

Blanco, I. 251

Bocskai, E. 156

Bonet, B. 146

Borawska, M.H. 134

Burus, I. 202

Büscher, U. 241, 357

Carbajal, A. 125

Chabchoub, S.E. 8

Chai, Y.-M. 151

Chauveau, P. 409

Chen, F. 357

Chida, K. 390

Cho, Y.-O. 235

Choi, J.-H. 151

Choi, M.-S. 151

Christophe, A.B. 335

Chung, H.-S. 398

Cole, D.E.C. 381

Collin, M. 228

Combe, C. 409

Combe, E. 281

Contaldo, F. 189
Cottel, D. 43

Cresi, F. 84

Cser, M.A. 156

Dallongeville, J. 43

D'Astous, M. 381

Decsi, T. 202

De Luis, J. 141

Domonkos, A. 307

Dosy, J. 381

Ducimetière, P. 43

Dudenhausen, J.W. 357

Dušinská, M. 118

Dyerberg, J. 61

El-Gendy, A. 343

El-Habibi, E.-S. 343

Elmadfa, I. 156, 169

Escudero, J.C.M. 141

Evroski, J. 381

Fauvel, J. 43

Feoli, A.M. 246

Ferrières, J. 43

Funke, S. 202

Fürst, P. 210

García-Arias, M.T. 125

García-Fernández, M.C. 125

García-Linares, M.C. 125

Gin, H. 409

Gomez, J.E. 329

Goñi, I. 369

Gonzalez Sagrado, M. 141

Gottschalk, S. 79

Granado, F. 251

Grasso, G. 84

Greiner, E. 307

Gupta, R. 221

Hahn, A. 103, 163

Hamdaoui, M.H. 8

Hayashi, T. 276

Hédhili, A. 8

Herreros, V. 141

Hong, S.-H. 398

Houti, I. 296

Howe, P.R.C. 67

Høy, C.-E. 16

Huang, P.-C. 321

Hukałowicz, K. 134
Ide, K. 79

Inaba, Y. 390

Ishihara, K. 348

Isik, Y. 259

Izaola, O. 141

Japan Idiopathic Pulmonary Fibrosis Study Group 390

Joo, G.-J. 151

Joseph, F., Jr. 79

Kalyoncu, M. 259

Kamal-Eldin, A. 169

Kang, M.-H. 36

Kanning, U. 263

Kärkkäinen, M. 228

Kaur, J. 221

Kaur, M. 221

Kempaiah, R.K. 314

Kersting, M. 414

Kim, B.-Y. 235

Kim, H.-M. 398

Kim, K.-R. 151

Kim, N.-H. 398

Klvanová, J. 118

Kobashi, G. 390

Korpela, R. 228

Koschizke, J.W. 103

Kovács, A. 202

Kovács, I. 156

Krajčovičová-Kudláčková, M. 118

Krawinkel, M.B. 109

Krüger, A.H. 246

Kudoh, S. 390

Lamberg-Allardt, C. 228

Larkin, T.A. 67

Lasseur, C. 409

Lee, I.-S. 151

Leitzmann, C. 103

Lima, L.A.M. 404

Lin, M.-H. 321, 374

Liu, S.-Y. 321, 374

Liu, Y.-C. 321, 374

Lu, S.-C. 321

Luis Román, D.A. de 141

Lund, S.P. 16

Mahmood, A. 221

Majchrzak, D. 156

Markiewicz, R. 134

Marosvölgyi, T. 202

\section{KARGER}

(c) 2004 S. Karger AG, Base

Fax + 41613061234

E-Mail karger@karger.ch

www.karger.com
Accessible online at: www. karger.com/anm 
Marra, M. 189

Masse, P.G. 381

Matsushima, R. 348

Meyer, B.J. 67

Minda, H. 202

Miyake, Y. 390

Mokuda, O. 276

Molnár, S. 202

Moussa, M.A.A. 329

Moussamih, S. 296

Mozsáry, E. 307

$\mathrm{Mu}, \mathrm{H} .16$

Murata, M. 348

Nagy, K. 307

Nakamoto, T. 79

Narva, M. 228

Navarro, F. 90

Nieto, S. 28

Novaes, L.C.G. 404

Novaes, M.R.C.G. 404

Oggero, R. 84

Ojha, S. 221

Okamoto, K. 390

Okazaki, R. 276

Okten, A. 259

Olmedilla, B. 251

Ooka, H. 276

Orešnik, A. 281

Owen, A.J. 67, 269

Pallauf, J. 109

Park, E. 36

Park, E.-J. 398

Pasanisi, F. 189

Patureau Mirand, P. 281

Pereira Bacurau, R.F. 90

Perez Castrillon, J.L. 141

Perlemoine, C. 409

Perry, C.T. 246

Perry, M.L.S. 246

Pirman, T. 281
Pons, A. 193

Poussa, T. 228

Qotbi, S. 296

Raffaitin, C. 409

Raila, J. 241, 357

Ramírez, G. 28

Refai, T.M.K. 329

Regöly-Mérei, A. 307

Rhee, I.-K. 151

Rhee, S.-J. 151

Rigalleau, V. 409

Roach, P.D. 269

Rodríguez, Y. 335

Rodriguez Amaral Almeida, A.L. 90

Roky, R. 296

Romaguera, D. 193

Romero, E. 141

Rotta, L.N. 246

Rust, P. 156

Sakamoto, N. 390

Sakamoto, Y. 276

Saldanha Aoki, M. 90

Sánchez-Muniz, F.J. 125

Sánchez-Vera, I. 146

Sanhueza, J. 28

Sano, Y. 348

Sanz, C. 146

Sasaki, S. 390

Savino, F. 84

Scalfi, L. 189

Schaffer, S. 109

Schlotzer, E. 263

Schweigert, F.J. 241, 357

Sehouli, J. 241

Shirai, N. 51, 95

Sichert-Hellert, W. 414

Silvestro, L. 84

Siqueira, E.M.A. 288

Sommer Hartvigsen, M. 16

Sørig Hougaard, K. 16
Souza, D.O. 246

Souza, E.M.T. 288

Souza, K.B. de 246

Souza, M.R. de 246

Souza, M.V. 404

Spodaryk, M. 215

Srinivasan, K. 314

Stekar, J. 281

Stender, S. 61

Suda, T. 390

Suzuki, H. 51, 95

Szabó, C. 307

Szász, M. 202

Sziklai-László, I. 156

Szórád, I. 307

Sztefko, K. 215

Tanaka, H. 276, 390

Tapsell, L.C. 67

Tauber, J.-P. 43

Tomasik, P.J. 215

Tur, J.A. 193

Uchida, M. 348

Valenzuela, A. 28

Valenzuela, V. 28

Vapaatalo, H. 228

Vaquero, M.P. 125

Viana, M. 146

Wagner, A. 43

Wagner, K.-H. 169

Waldmann, A. 103

Washio, M. 390

Wirth, K. 357

Witkowska, A.M. 134

Wolters, M. 163

Xu, X. 16

Yazdani, M. 79

Yokoyama, T. 390 